\title{
Repensar la formación inicial docente desde una dimensión socioemocional
}

\author{
López-López, Verónica; Lagos San Martín, Nelly \\ Repensar la formación inicial docente desde una dimensión socioemocional \\ Revista Educación, vol. 45, núm. 1, 2021 \\ Universidad de Costa Rica, Costa Rica \\ Disponible en: http://www.redalyc.org/articulo.oa?id=44064134002 \\ DOI: https://doi.org/10.15517/revedu.v45i1.41464
}

Esta obra está bajo una Licencia Creative Commons Atribución-NoComercial-SinDerivar 3.0 Internacional. 


\title{
Repensar la formación inicial docente desde una dimensión socioemocional
}

\author{
Rethinking Initial Teacher Training from a Socioemotional Dimension
}

Verónica López-López

Universidad de Concepción, Chile

veronicalopez@udec.cl

(iD http://orcid.org/0000-0002-8324-6692

Nelly Lagos San Martin

Universidad del Bio-Bio, Chile

nlagos@ubiobio.cl

iD http://orcid.org/0000-0002-2029-5219
DOI: https://doi.org/10.15517/revedu.v45i1.41464 Redalyc: http://www.redalyc.org/articulo.oa?id=44064134002
Recepción: 01 Mayo 2020

Aprobación: 07 Agosto 2020

\section{RESUMEN:}

El artículo tiene por objetivo ofrecer una reflexión sobre la formación docente en un contexto de profundos cambios y transformaciones sociales que han interpelado a la formación inicial del profesorado en su labor pedagógica. Formación que debiera ser repensada en consonancia con estos cambios en donde se valore las competencias socioemocionales como la autoconciencia, la autogestión, la conciencia del otro, las habilidades sociales y la toma de decisiones responsables, pues una competencia no adquirida no se puede enseñar. Este análisis se plantea considerando que el profesorado es un profesional capaz de integrar saberes y valores, pues enseña en un contexto socialmente construido con desigualdades y vulnerabilidad económica y social, cuyo rol es ser un mediador entre el saber y los procesos de apropiación del estudiantado. De ahí la necesidad de proponer una discusión en torno a esta temática.

Palabras ClaVe: Educación, Pedagogía, Formación docente, Competencias del profesorado.

\section{Abstract:}

This study reflects on Teacher Training within a context of profound social change and transformation which challenges initial teacher training and pedagogical endeavors. Teacher Training must be reevaluated and better aligned to such changes where selfawareness, self-management, awareness of the other, social skills and responsible decision-making must be valued as acquired skills that cannot be taught. Teachers should be able to integrate both knowledge and values within the socially constructed context where they teach and, at times, ridden with inequalities, economic constraints and social vulnerabilities. Their role is, decidedly, one as mediator of knowledge and a student appropriation process of that knowledge and for which further discussion is required to better understand this concept.

KEYWORDs: Education, Pedagogy, Teacher Training, Teacher Competencies.

\section{INTRODUCCIÓN}

Este artículo propone una reflexión acerca de la formación inicial docente en Chile desde una dimensión socioemocional debido a que las personas educadores son las profesionales responsables de la mediación, construcción de la conciencia y la producción de la subjetividad (Giroux, 2001); y por qué en la sociedad actual los sistemas educativos han sido interpelados por nuevos paradigmas y competencias profesionales, los cuales se basan en la idea de que una competencia que no se ha adquirido no se puede enseñar, como tampoco es posible la calidad en la enseñanza ante la ausencia del bienestar del cuerpo docente (Bassett, Haldenby, Tanner y Trewhitt, 2010).

Este proceso de cambio y transformación sociohistóricos es el resultado de las demandas sociales, reflexiones de una comunidad científica y de la reformulación de los estándares establecidos, lo cual busca 
responder a los criterios de calidad exigidos, acorde con el nuevo escenario sociocultural que atraviesan los estados, donde se han propuesto enfoques constructivistas, plasmados en las reformas educativas de distintos países (Ávalos, 2014; Tedesco, 1995) y que ha implicado replantear el sentido que tiene la educación y repensar su legado cultural, los valores y la sociedad que desea transmitir; en donde el profesorado será quien asuma nuevos roles y desarrolle nuevas competencias en un aula caracterizada por su vulnerabilidad y diversidad cultural (Ferrada, Villena y Turra, 2015).

Además, se ha observado que variadas disciplinas, como el pensamiento filosófico, pedagógico, psicológico, sociológico, antropológico y biológico, sugieren que, para aprender y desarrollarse, el ser humano debería tener conocimiento, regulación y autonomía de sus emociones, así como también comprensión de las emociones de las demás personas (Maturana, 1991; UNICEF, 2014; UNESCO, 2016). No obstante, este proceso se encuentra necesariamente vinculado con la formación del profesorado (Ávalos, 2014; Echeita, 2017), pues el rol del grupo docente es relevante en la construcción de sociedades democráticas, puesto que aporta la manera apropiada para resolver conflictos, establecer relaciones interpersonales equilibradas y desarrollar miradas de carácter inclusivo con respeto a la diversidad cultural.

\section{1.- La formación del profesorado en un contexto de diversidad y cambios sociales.}

La formación de docentes posee constantes y múltiples reflexiones, y análisis que se enmarcan bajo las denominadas reformas educativas que impulsan la renovación curricular y pedagógica. En la última década han sido varios los estudios acerca de esta temática en el mundo, los cuales se han abordado con enfoques distintos. Algunos estudios han destacado la relevancia de los conocimientos (Shulman, 1987), saberes (Tardif, 2001) o competencias (Perrenoud, 2001) necesarias en la formación docente (Puentes, Aquino y Neto, 2009). Otros, asimismo, han planteado la necesidad de subrayar el desarrollo de habilidades (Novoa, 2009) y la ética en la profesión docente (Sockett, 1994) que tome en consideración la identidad de la docencia (Korthagen, 2004).

En este sentido, la formación inicial docente en Chile, como en el contexto latinoamericano, se ha ido renovando en concordancia con las exigencias de las reformas educativas llevadas a cabo desde la década de los noventa hasta la fecha (Ferrada, Turra y Villena, 2013). Es a partir de esta década y en las dos siguientes, que se han situado y desarrollado variadas medidas cuyo propósito ha sido mejorar, rediseñar y fortalecer la calidad de la Formación Inicial Docente. En directa relación con lo recién mencionado, entre las principales medidas aplicadas se destacan: el Proyecto de Fortalecimiento de la Formación Inicial Docente (1997); el mejoramiento de la educación superior orientado específicamente a las pedagogías; la Acreditación Obligatoria de las Carreras de Pedagogía (2006); el Programa de Fomento a la Calidad de la Formación Inicial Docentes, Inicia (2008); los Convenios de Desempeño para mejorar los procesos de Formación Inicial Docente (FID, 2011); los estándares orientadores para las Carreras de Pedagogía (2011-2012); el Programa de Acompañamiento y Acceso Efectivo a la Educación Superior (2015) y actualmente la Ley del Sistema de Desarrollo Profesional Docente (2016).

Todas las medidas para mejorar, rediseñar y fortalecer la calidad de la Formación Inicial Docente son de responsabilidad de las instituciones de educación superior en Chile y se estructuran con base en un desempeño homogéneo, es decir, se forma al futuro profesorado para atender pedagógicamente a un/a estudiante promedio, perteneciente a la cultura occidental pensada de manera generalista, desconociendo la diversidad en estilos de aprendizaje y contextos culturales existentes en el país (Ferrada, Turra y Villena, 2013).

En total contraposición con la visión generalista, tanto del contexto como del estudiantado; Delors (1996), en su Informe de la Unesco, da importancia a la diversidad en cuanto a la función de las emociones y enfatiza en la necesidad de educar la dimensión emocional integrada a la cognitiva, pluralizando la enseñanza en vez de acotándola a un/a estudiante promedio y estrategias unidimensionales. Relacionado con esto 
también, la Declaración de Bolonia (citado en Montero, 2010), la cual resalta el valor de la educación en términos de adquisición de competencias, entre ellas las competencias socioemocionales y el proyecto Tuning (González y Wagenaar, 2003), financiado por la Comisión Europea en el marco del Programa Sócrates, permiten replantearse la excesiva racionalidad pedagógica dominante y posicionarse desde una pedagogía más vinculada al diálogo intercultural y crítica reflexiva (Carmona, 2008).

Los cambios y reformas que se han hecho en la Formación Inicial Docente tratan de formar un tipo de docente que integre los saberes con las experiencias, así como también que fortalezca la conexión con la vida cotidiana de las personas, vinculadas a su contexto personal, y que en su proceso de formación construya dimensiones epistemológicas, políticas y axiológicas, requeridas para ello. Es por esto, que hoy es el momento de diferenciar el trasfondo de lo que efectivamente debe ser renovado: la racionalidad que integre lo emocional (Sánchez, 2012).

Esta nueva concepción construida sobre la base de los cambios epistemológicos del siglo XX, busca superar esta desconexión entre lo enseñado en la formación y lo requerido en el rol docente, con el fin de proporcionar herramientas concretas que le permitan superar las tensiones que se generan en la fase de iniciación, en donde el profesorado experimenta el desajuste entre la formación y las demandas a su desempeño (Ávalos, 2014). Debido a esto, es importante que el proceso formativo no sea influenciado por presiones ideológicas o de grupos de poder, sino que se centre en un aprendizaje orientado hacia una autonomía del pensamiento (Marsillac y Gastahlo, 2012).

El estudio de Hascher y Hagenauer (2016) señala que en un/a docente no solo se asientan los conocimientos adquiridos durante su proceso de formación, sino que además se inscriben las competencias socioemocionales que ha logrado experimentar durante su formación (Palomero, 2009). Esto implicaría, entonces, que la integración de lo socioemocional va más allá de enseñarlo en el aula como contenido o usarlo metodológicamente para educar, sino también un proceso que rescata lo ontológico, la vivencia de cada docente y su labor. Por lo mismo, no pueden seguir formándose docentes reproductores y transmisores de conocimientos establecidos como verdades absolutas, muy por el contrario, deben ser formados con capacidad crítica y reflexiva, de tal modo que les permita enfrentar las incertidumbres y las divergencias, elementos esenciales que rescatan el contexto y que conviven en el desarrollo (Imbernón, 1994; Kemmis, 1993).

En el fondo, se trata de desarrollar una pedagogía que reconozca el sentido de la pluralidad, diversidad y la subjetividad del otro, así como de sí mismo. De allí que la formación del profesorado tenga que orientarse hacia la integración de saberes y valores para la construcción humana, además de asociarse necesariamente a un diálogo intersubjetivo, con contenidos emergentes y nuevos ámbitos culturales que enriquezcan las experiencias del cuerpo docente. Debido a esto, resulta necesario replantear su formación en Chile y considerarla un eje fundamental para que se comiencen a vincular las emociones de manera formal, no solo porque haya demostrado ser relevante en otros ámbitos (éxito académico, disminución de la violencia, mejora de la calidad de vida), sino porque a pesar de la importancia de estas competencias, el personal docente ha reportado escasez de estrategias del aprendizaje socioemocional en el aula (Berger et al., 2009; Casassus, 2015).

Es así como las competencias socioemocionales no debieran relegarse a los currículos de las carreras de pedagogía ni desterrarlas a los espacios ocultos, donde dependerían de las voluntades y serían objeto de un tratamiento poco riguroso, y por tanto, estarían sometidas al albur de la intuición y la improvisación de cada docente (Vaello, 2005). Se trata de desarrollar en el futuro profesorado una capacidad reflexiva que se conciba como la manera de articular conocimientos y creencias, y analice sus fundamentos, además de su posible adecuación a un determinado contexto (Schön, 1992). Por lo tanto, la formación no solo debiese circunscribirse a competencias genéricas, sino ampliarse a competencias socioemocionales (Villa y Poblete, 2011). 
En este sentido, y tomando en cuenta las dificultades planteadas en la Formación Inicial Docente, el proceso de aprendizaje debiera incorporar el desarrollo de habilidades socioemocionales. Para esto, es preciso destacar lo señalado por el Artículo $2^{\circ}$ de la Ley General de Educación en Chile (Ley 20.370), la cual ya lo establece al indicar que la finalidad de la educación es lograr el desarrollo espiritual, ético, moral, afectivo, intelectual, artístico y físico (Biblioteca del Congreso Nacional de Chile, 2009).

\section{2.- Nuevos desafíos en la formación inicial docente.}

La sociedad actual ha interpelado a los sistemas educativos con el fin de requerir nuevos paradigmas o enfoques, además de profesionales competentes las cuales planteen propuestas reales y viables frente a los problemas complejos y dinámicos que se presentan en una diversidad de contextos de vulnerabilidad social. Proceso que se encuentra asociado a transformaciones sociohistóricas como el resultado de las demandas sociales, investigaciones educativas, reflexiones de una comunidad científica y de la reformulación de los estándares establecidos; orientados a responder criterios de calidad acordes con el nuevo escenario sociocultural de los estados.

Este nuevo escenario ha implicado replantear el sentido que tiene la educación sobre el legado cultural, los valores y el tipo de sociedad que se desea transmitir (Ávalos, 2014; Tedesco, 1995), y por ende, repensar la formación de docentes para asumir nuevos roles y desarrollar nuevas competencias en su práctica educativa, en un aula que se caracteriza por su vulnerabilidad y diversidad cultural (Ferrada, Villena y Turra, 2015). Esto también implica una transformación en el cumplimiento de criterios de calidad como el clima de convivencia escolar, la formación ciudadana, equidad de género, los hábitos de vida saludable y la retención escolar; los cuales fueron establecidos por el Consejo Nacional de Educación de Chile.

Todo este proceso requiere de una reflexión pedagógica (Schön, 1992) y de un cambio de paradigma en la formación docente que integre, gradualmente, en el currículo de los futuros profesores, la dimensión socioemocional con la cognitiva como un eje transversal, de manera que en la transferencia y relación docenteestudiante no sea dejada a la voluntad del profesorado al momento de enseñar las formas más adecuadas de cómo resolver conflictos y construir relaciones interpersonales desde una mirada inclusiva e intercultural; es decir, que el cuerpo docente en su formación inicial tome conciencia de la importancia de las competencias socioemocionales para superar los obstáculos, prejuicios y la discriminación (Bandura, 2003; Blanco, 2006; CASEL, 2020; Nussbaum, 2012; Quintriqueo, Quilaqueo, Gutiérrez y Peña, 2015).

Pero, ¿por qué se querría replantear la formación docente desde una dimensión socioemocional? Estos debates teóricos han sido planteados por la sociología y antropología a lo largo del siglo XX en los cuales se incorporaron las emociones como categoría de análisis sociohistórico y como objeto de estudio cultural, surgiendo una categoría relacional, lo cual permite ampliar la comprensión sobre la forma en que el individuo y la sociedad se relacionan. Fue así como generaron un cambio en su epistemología y le permitió tomar distancia de las disciplinas identificadas con el paradigma positivista (Bolaños, 2016; Suárez, 2016).

Por consiguiente, esta categoría de análisis relacional de las emociones, se comienza a estudiar en la escuela, debido a que es un espacio donde confluyen y se interconectan las emociones de los diferentes actores sociales convirtiéndose en un tema central, pues estas competencias son las que acompañan el desarrollo del estudiantado a la adultez en su ámbito familiar, social y laboral (Bassi, Busso, Urzua y Vargas, 2012; Casassus, 2015; Extremera, Rey y Pena, 2016; Mariño, Pulido y Morales, 2016).

Según Repetto y Pena (2010), existen diversas investigaciones las cuales han mostrado que estudiantes con mayor éxito académico poseen mejores niveles de competencias emocionales. Lopes y Salovey (2004) destacan que existen trabajos que prueban cómo la aplicación de algunos programas para el desarrollo de estas competencias incrementa no solo el proceso de aprendizaje y el éxito académico del alumnado, sino que también favorece la integración social. De esta manera, estos programas comienzan a plantear la necesidad de desarrollar una educación emocional, la cual de acuerdo con la definición de Bisquerra (2003) es "el conjunto 
de conocimientos, capacidades, habilidades y actitudes necesarias para comprender, expresar y regular de forma apropiada los fenómenos emocionales" (p.21).

\section{3.- Relevancia de las competencias socioemocionales en el profesorado.}

La profesión docente es una labor altamente compleja y exigente, esta requiere para su desempeño de múltiples y variadas competencias personales y profesionales, entre las cuales se incluyen los conocimientos teóricos y pragmáticos propios de la disciplina. La labor pedagógica se encuentra vinculada a un aula; un lugar de encuentro, no solo de saberes, que está cargada de emociones y sentires. Un espacio socioeducativo que culturalmente es diverso y, en ocasiones, vulnerable social y afectivo (Ferrada, Turra y Villena, 2013).

Los contextos educativos son escenarios donde se debiera integrar las competencias socioemocionales, por ser cada docente un/a profesional responsable de la mediación pedagógica y porque diversos estudios en el campo del desarrollo evolutivo relacionan una alta habilidad en competencias socioemocionales con mayor bienestar, climas positivos de trabajo y rendimiento escolar o laboral, mientras que una baja habilidad en estas competencias conlleva a dificultades en el ámbito personal, social, académico y profesional (Damon, Lerner y Eisemberg, 2006).

Por lo anterior, resulta importante que el profesorado cuente con habilidades socioemocionales, puesto que juega un papel fundamental en el modelado y la enseñanza de habilidades socioemocionales (Vaello, 2005), pues en el aula es un valioso instrumento de influencia social, en tanto permiten generar cambios en el pensamiento, las actitudes y los comportamientos de los demás y aportan recursos para afrontar retos de la vida profesional, lo cual permite superar situaciones complicadas mediante el aporte de competencias como la resiliencia, el autocontrol, que se encuentran en la base de cualquier logro profesional (Vaello, 2005; Zahonero y Martín, 2012).

Sin embargo, si estas competencias no han sido adquiridas por el profesorado se dificulta su enseñanza (Bassett, Haldenby, Tanner y Trewhitt, 2010), así, surge la necesidad de repensar la formación inicial docente desde una dimensión socioemocional. Esto implica revisar los currículos de las carreras de pedagogía, para, mediante los procesos de rediseño, integrar en la formación el desarrollo de las competencias socioemocionales de los futuros maestros, para abordar la formación personal del cuerpo docente, toma responsable de decisiones, conciencia de uno mismo, autorregulación, conciencia social y habilidades de relación (CASEL, 2020).

Es por esto, que la mayoría de los autores coinciden en que un/a docente debe tener, además de conocimientos didácticos y el uso de nuevas tecnologías, destrezas para comunicarse y relacionarse positivamente con los diferentes actores de la comunidad educativa. Competencias vinculadas a liderar y manejar el aula como saber trabajar en equipo y tomar decisiones responsables y éticas para la resolución de problemas en el centro educativo (Palomera, Briones y Gómez, 2017).

Todos estos factores son fundamentales para el desempeño profesional del profesorado, porque permiten potenciar en estudiantes valores humanos como la empatía, la colaboración, la responsabilidad social, la ética, entre otros aprendizajes que contribuirían en la manera de resolver de conflictos y desarrollar una mirada inclusiva, la cual abarque la diversidad cultural y las perspectivas de género, entre otros tópicos (Herrera, Buitrago, Lorenzo y Badea, 2015). En este sentido, han surgido propuestas actuales, en consonancia con los cambios socioculturales y la necesidad de que cada docente desarrolle nuevas competencias, como es la introducción de competencias intrapersonales relacionadas, por ejemplo, con el autoconocimiento o la estima personal (López y Zabala; 2012) y programas de educación propuestos por el modelo de Collaborative for Academic, Social, and Emotional Learning (CASEL, 2020). Es posible repensar la formación inicial docente desde una dimensión socioemocional, integrando gradualmente en el currículo nacional las competencias socioemocionales anteriormente descritas. 


\section{Discusión}

Las transformaciones socioculturales presentes en cada época interpelan y obligan a la educación a reflexionar y replantearse críticamente el sentido y la finalidad de esta. En esta dirección, se propone la necesidad de repensar la formación inicial docente desde la dimensión socioemocional como eje fundamental, debido a que, si las competencias socioemocionales no han sido adquiridas, se dificulta su enseñanza. Se desprende de esto porque al constituirse las emociones como una categoría de análisis relacional, se comienzan a estudiar en la escuela, debido a que es un espacio donde confluyen y se interconectan las emociones de los diferentes actores sociales, convirtiéndose en un tema central, pues estas competencias son las que acompañan el desarrollo del estudiantado a la adultez en los ámbitos familiar, social y laboral.

Es necesario comprender que el proceso pedagógico se expande hacia nuevos espacios culturales, los cuales van acompañados de significados afectivos y emocionales. Este proceso, requiere de nuevos modos de análisis para la redefinición en su formación y de las políticas educativas que la pedagogía como disciplina instala, producto del colmado de significados vinculados al saber pensar, saber decir y saber actuar. Saberes que son cruciales en la proyección de la vida social y laboral, como es la habilitación ética y ciudadana y las competencias socioemocionales. Estas últimas vinculadas a la autoconciencia, la autogestión, la conciencia del otro, las habilidades sociales y la toma de decisiones responsables y las que debieran ser integradas en el currículo nacional de manera gradual como un eje en la formación, comenzando desde una dimensión personal a una social en la cual se incluya la toma de decisiones responsables.

\section{Conclusiones}

Este cambio epistémico de revaloración de la educación socioemocional, generado desde fines del siglo XX, es una de las consideraciones que debe profundizar la pedagogía contemporánea. Esto implica una doble consideración: por un lado, incorporarla a la Formación Inicial Docente; y por otro, reflejarse en la práctica docente. Respecto de la Formación Inicial Docente, no se la puede seguir olvidando en los currículos de las carreras de pedagogía en el país y por lo tanto, dejarla al libre albedrío como tradicionalmente se ha hecho; esto debido a que la pedagogía se convierte en una herramienta indispensable para la formación de una ciudadanía con contenido crítico y trascendente, es decir, con un desarrollo de una conciencia crítica colectiva.

Todo esto redunda en la necesidad de que la educación y en especial el profesorado, transfieran conocimientos con estrategias no solo cognitivas, sino emocionales en el aula y en el centro educativo. Además, tal como se mencionó, en los acápites anteriores, docentes y estudiantes alfabetizados emocionalmente, gestionan y administran de mejor manera sus emociones; no se trata de enseñarlas o hacerlo desde la emocionalidad, sino de educar con ella.

Finalmente, llama la atención, que aún en pleno siglo XXI el conocimiento, cuerpo externo de informaciones, el cual se aborda en el aula pareciera que fuera independiente de los seres humanos y estuviera más bien centrado en un conocimiento que llega a ser no solo contable y mensurable, sino también impersonal. Debido a esto, repensar la formación inicial de docentes desde una dimensión socioemocional es un desafío, el cual debiera abordarse en instancias formales y periódicas de evaluación, junto con la revisión del plan de estudios de las carreras de pedagogía, que resulta ser una de las debilidades planteadas por la Comisión Nacional de Acreditación (CNA-Chile), pues no existen mecanismos de evaluación de la pertinencia de las actualizaciones, los cuales consignen un currículo que aborde la labor pedagógica e incluya, gradualmente, como eje transversal el desarrollo de la conciencia emocional, la regulación y modulación de las emociones desde una mirada crítica y que esté [el currículo] vinculado a la formación de valores ciudadanos como el respeto, la tolerancia y la inclusión, debido a que una competencia no adquirida no se puede enseñar, como tampoco es posible la calidad en la enseñanza ante la ausencia del bienestar del profesorado. 


\section{Agradecimientos}

Este trabajo se desarrolló en el contexto del doctorado en Educación, apoyado por el Grupo de Investigación de Desarrollo Emocional y Cognitivo para el Aprendizaje (Gidecap), Código: DIUBB 195623/VC.

\section{ReFERENCIAS}

Ávalos, B. (2014). La formación inicial docente en Chile: tensiones entre políticas de apoyo y control. Estudios Pedagógicos (Valdivia), 40 (Especial), 11-28. doi: http://dx.doi.org/10.4067/S0718-07052014000200002

Bandura, A. (2003). On the psychosocial impact and mechanisms of spiritual modeling. International Journal for the Psychology of Religion, 13(3), 167-173.

Bassi, M., Busso, M., Urzua, S. y Vargas, J. (2012). Desconectados: habilidades, educación en América Latina. New York: BID Educación.

Berger, C., Milicic, N., Alcalay, L., Torretti, A., Arab, M. y Justiniano, B. (2009). Bienestar socio-emocional en contextos escolares: la percepción de estudiantes chilenos. Estudios sobre Educación, 17, 21-43.

Bassett, D., Haldenby, A., Tanner, W., y Trewhitt, K. (2010). Every teacher matters. London: Reform.

Biblioteca del Congreso Nacional de Chile (2009). Ley General de Educación en Chile. Recuperado de https://www .leychile.cl/Navegar?idNorma $=1006043$

Bisquerra, R. (2003). Educación emocional y competencias básicas para la vida. Revista de investigación, 21(1), 7-43.

Blanco, R. (2006). La equidad y la inclusión social: uno de los desafíos de la educación y la escuela hoy. Revista Electrónica Iberoamericana sobre Calidad, Eficacia y Cambio en Educación, 4(3), 59-68.

Bolaños, L. (2016). El estudio socio-histórico de las emociones y los sentimientos en las Ciencias Sociales del siglo XX. Revista de Estudios Sociales, 55, 178-191.

Carmona, M. (2008). Hacia una formación docente reflexiva y crítica: Fundamentos filosóficos. Revista de teoría y didáctica de las ciencias sociales, 13, 125-146.

Casassus, J. (2015). La educación del ser emocional. Santiago de Chile: editorial Cuarto Propio.

Collaborative for Academic, Social, and Emotional Learning [CASEL] (2020). Core SEL Competencies. Recuperado de https://casel.org/core-competencies/

Damon, W., Lerner, R. y Eisenberg, N. (Eds.). (2006). Handbook of Child Psychology: Volume Three: Social, Emotional, and Personality Development. New York: Wileyy Sons.

Delors, J. (1996). La Educación Encierra un Tesoro, Informe de la Comisión de la Unesco para la Educación del siglo $X X I$. Madrid: Santillana.

Echeita, G. (2017). Educación inclusiva. Sonrisas y lágrimas. Aula Abierta, 46(2), 17-24.

Extremera, N., Rey, L. y Pena, M. (2016). Educadores de corazón. Inteligencia emocional como elemento clave en la labor docente. Revista Padres y Maestros, (368), 65-72. doi: https://doi.org/10.14422/pym.i368.y2016.011

Ferrada, D., Turra, O. y Villena, A. (2013). Currículum transformador de formación inicial para profesores en contextos de vulnerabilidad social. Cadernos de Pesquisa, 43(149), 642-661. doi: https://doi.org/10.1590/S01 00-15742013000200013

Ferrada, D., Villena, A. y Turra, O. (2015). Transformar la formación: las voces del profesorado. Santiago: RIL Editores. Giroux, H. (2001). Los profesores como intelectuales transformativos. Docencia, 6(15), 60-66.

González, J. y Wagenaar, R. (2003). El Proyecto Tuning Educational Structures in Europe. Bilbao: Universidad de Deusto.

Hascher, T. y Hagenauer, G. (2016). Openness to theory and its importance for pre-service teachers' self-efficacy, emotions and classroom behaviour in the teaching practicum. International Journal of Educational Research, 77 , 15-25. doi: https://doi.org/10.1016/j.ijer.2016.02.003 
Verónica López-López, et aL. Repensar la formación inicial docente deSDe una dimensión SOCiOEMOCiO...

Herrera, L., Buitrago, R., Lorenzo, O. y Badea, M. (2015). Socio-emotional intelligence in Colombian children of primary education. An analysis in rural and urban settings. Procedia - Social and Behavioral Sciences, 203, 4-10. doi: https://doi.org/10.1016/j.sbspro.2015.08.251

Imbernón, F. (1994). La Formación y el Desarrollo Profesional del Profesorado. Hacia una nueva cultura profesional. Barcelona: Graó.

Kemmis, S. (1993). El curriculum: más allá de la teoría de la reproducción. Madrid: Morata.

Korthagen, F. (2004). In search of the essence of a good teacher: towards a more holistic approach in teacher education. Teaching and Teacher Education, 20(1), 77-97. doi: https://doi.org/10.1016/j.tate.2003.10.002

López, I. y Zabala, J. (2012). La competencia emocional en los currículos de formación inicial de los docentes. Un estudio comparativo. Revista de Educación, 357, 467-489. doi: https://doi.org/10.4438/1988-592X-RE-2011 $-357-069$

Lopes, N. y Salovey, P. (2004). Toward a broader education. En H. Walberg, M., Wang, R. Zins y P. Weissberg (Eds.), Building school success on social and emotional learning (pp. 79-93). New York: Teachers and College press.

Mariño, L., Pulido, Ó. y Morales, L. (2016). Actitud filosófica, infancia y formación de maestros. Praxisy Saber, 7(15), 81-101. doi: https://doi.org/10.19053/22160159.v7.n15.2016.5724

Marsillac, A. y Gastalho, P. (2012). Desencontros entre uma prática crítica em psicologia e concepções tradicionais em educação. Revista Semestral da Associação Brasileira de Psicologia Escolar e Educacional, 16(1), 153-160.

Maturana, H. (1991). Emociones y Lenguaje en Educación y Política. Santiago: Dolmen Ediciones.

Montero, M. (2010). El Proceso de Bolonia y las nuevas competencias. Tejuelo, 9, 19-37.

Novoa, A (2009). Profesores: ¿el futuro aún tardará mucho tiempo? En C. Vélaz y D. Vaillant (Ed.), Aprendizaje y desarrollo profesional docente (pp. 49-55). Madrid: Fundación Santillana

Nussbaum, M. (2012). Crear capacidades. Propuestas para el desarrollo humano. Barcelona: Paidós

Palomera, R., Briones, E. y Gómez, A. (2017). Diseño, desarrollo y resultados de un programa de Educación SocioEmocional para la formación de docentes a nivel de Grado y Postgrado. Contextos Educativos. Revista de Educación, (20), 165-182. doi: https://doi.org/10.18172/con.2988

Palomero, P. (2009). Desarrollo de la competencia social y emocional del profesorado: una aproximación desde la psicología humanista. Revista Electrónica Interuniversitaria de Formación del Profesorado, 12(2), 145-153.

Perrenoud, P. (2001). La formación de los docentes en el siglo XX. Revista de Tecnología Educativa, 14(3), 503-523.

Puentes, R., Aquino, O., y Neto, A. (2009). Profissionalização dos professores: conhecimentos, saberes e competências necessários à docência. Educar em Revista, 34, 5-20.

Quintriqueo, S., Quilaqueo, D., Gutiérrez, M. y Peña, F. (2015). Enseñanza de Historia, Geografía y Ciencias Sociales: hacia una Perspectiva Intercultural. Temuco: Editorial Universidad Católica de Temuco.

Repetto, E. y Pena, M. (2010). Las Competencias Socioemocionales como Factor de Calidad en La Educación. Revista Iberoamericana sobre Calidad, Eficacia y Cambio en Educación, 8(5), 82-95.

Sánchez, J. (2012). La formación docente. Temas, debates y escenarios de prioridades. Acción pedagógica, 21(1), 58-63.

Schön, D. (1992). La formación de profesionales reflexivos. Barcelona: Paido\#s.

Shulman, L. (1987). Knowledge and teaching: foundations of the new reform. Harvard Educational Review, 57(1), 1-22. doi: https://doi.org/10.17763/haer.57.1.j463w79r56455411

Sockett, H. (1994). The Moral Base for Teacher Professionalism. New york: Teachers College Press.

Suárez, D. (2016). Ciencia y emociones: ¿responde la exclusión de la emotividad en la investigación científica a un prejuicio androcéntrico? en Tábano. Revista de filosofía, Facultad de Filosofía y Letras, Universidad Católica Argentina, (12), 71-90.

Tardif, M. (2001). Los saberes del docente y su desarrollo profesional. Madrid: Narcea.

Tedesco, J. (1995). El nuevo pacto educativo. Madrid: Grupo Anaya SA.

UNESCO (2016). Recomendaciones de Politicas Educativas en América Latina en base al Terce. Santiago: Oficina Regional de Educación para América Latina y el Caribe, Orealc/Unesco. 
UNICEF (2014). El Papel de la Educación en la formación del Bienestar Subjetivo para el Desarrollo Humano. Una revisión al caso chileno. Santiago: PNUD

Vaello, J. (2005). Habilidades sociales en el aula. Madrid: Santillana.

Villa, A. y Poblete, M. (2011). Evaluación de competencias genéricas: Principios, oportunidades y limitaciones. Bordón, 63, 147-170.

Zahonero, A. y Marti\#n, M. (2012) Cómo organizar espacios y tiempos en la universidad, centrados en las personas. Madrid: Pira\#mide-Anaya.

CC BY-NC-ND 